\title{
PROYECTO DE APRENDIZAJE TUTORADO: SESIÓN DE PSICOMOTRICIDAD. BUENA PRÁCTICA PARA MAESTROS- PSICOMOTRICISTAS DE ESCUELAS DE INFANTIL Y PRIMARIA DE BARCELONA EN COLABORACIÓN CON LA UAB
}

Teaching Project: Psychomotor session. Good practise for psychomotor teacher of preschool and primary schools of Barcelona in collaboration with UAB

Projeto de Aprendizagem Tutelado: sessão de psicomotricidade. Boas práticas para professores psychomotricians de ensino infantil e básico em Barcelona em colaboração com a UAB

\section{Carolina Nieva Boza (1)}

\section{Lurdes Martínez Mínguez (2)}

(1) Universitat Autònoma de Barcelona, España. Teléfono: +34 935812674. Correo electrónico: carolina.nieva@uab.cat

(2) Universitat Autònoma de Barcelona, España. Correo electrónico: lurdes.martinez@uab.cat

Este estudio se ha llevado a cabo dentro del proyecto de $\mathrm{I}+\mathrm{D}+\mathrm{i}$ : “La competencias docentes en la formación inicial del profesorado de educación física”. Convocatoria de noviembre de 2013 del Programa Estatal de Investigación, Desarrollo e Innovación Orientada a los Retos de la Sociedad, en el marco del Plan Estatal de Investigación Científica y Técnica y de Innovación 2013-2016. Referencia: EDU 2013-42024-R. Duración: 3 años (2014-2016).

Millora i innovació en la formació psicomotriu inicial de mestres. Anàlisi de mecanismes de vinculació d'aprenentatges teòrics amb pràctica real a les escoles fent ús de metodologies actives i avaluació formativa. Convocatòria d’Ajuts de recerca en millora i innovació en la formació inicial de mestres per a les titulacions impartides a les universitats participants en el Programa de Millora i Innovació en la Formació de Mestres (ARMIF 2014), de l'Agència de Gestió d'Ajuts Universitaris i de Recerca (AGAUR). Referencia: 2014-ARMIF-00031.

Proyecto de Aprendizaje Tutorado: Sesión de psicomotricidad. Buena práctica para maestrospsicomotricistas de escuelas de infantil y primaria de Barcelona en colaboración con la UAB 


\title{
Resumen
}

En la presente comunicación se expone una investigación realizada en la asignatura “Educación Psicomotriz en los centros de Educación Infantil”, del Grado de Educación Infantil de la Universidad Autónoma de Barcelona (UAB), donde se lleva a cabo un Proyecto de Aprendizaje Tutorizado (PAT), relacionando universidad, escuelas y estudiantes. Dicho estudio tiene como objetivo analizar las percepciones del docente psicomotricista de escuela en relación a los PAT, compartiendo con el profesorado universitario la tutorización académica, en una asignatura de formación inicial. Los resultados más significativos se refieren a aspectos organizativos y pedagógicos, sobre todo a nivel de información, planificación y evaluación. Las conclusiones muestran que el PAT es una herramienta clave para la adquisición de un aprendizaje significativo y competencial del estudiantado.

Palabras clave: Proyecto de Aprendizaje Tutorado; Buena práctica; Formación inicial profesorado; Educación psicomotriz; Educación infantil

\begin{abstract}
This paper explains a research carried out in the subject "Psychomotor Education in preschool education centers”, of Pre-school Education Degree of the Autonomous University of Barcelona (UAB), where is carried out a Teaching Project, connecting university, schools and students. This research aims to analyze the perceptions of psychomotor teachers of schools regarding the Teaching Projects, shared with the university lecturer the tutoring, in an Initial teacher education. The most significant results refer to organizational and pedagogical aspects, especially at the level of information, planning and evaluation. The conclusions of the research show that Teaching Project is a key tool for the acquisition of meaningful and competent learning of the students.
\end{abstract}

Keywords: Teaching Project; Good practise; Inicial teacher education; Psychomotor education; Pre-school education

\section{Resumo}

Nesta comunicação uma investigação sobre o assunto "psicomotora Educação em préescolas" do grau de Educação Infantil da Universidade Autónoma de Barcelona (UAB),

Proyecto de Aprendizaje Tutorado: Sesión de psicomotricidad. Buena práctica para maestrospsicomotricistas de escuelas de infantil y primaria de Barcelona en colaboración con la UAB 
onde é realizado um projeto de aprendizagem autorizado exposta (PAT ), ligando universidades, escolas e alunos. Este estudo tem como objetivo analisar as percepções do professor psicomotricista Em relação tutelados Projetos de Aprendizagem (PAT), compartilhados com a tutoria acadêmica do corpo docente da universidade, em um curso de formação inicial. Os resultados mais significativos dizem respeito a aspectos organizacionais e pedagógicas, especialmente em termos de informação, planejamento e avaliação. Os resultados mostram que o PAT é uma ferramenta chave para a aquisição de uma aprendizagem do aluno significativa e habilidade.

Palavras-chave: Explicações Projeto de Aprendizagem; Boas práticas; faculdade formação inicial; psicomotricidade; Educação infantil

\section{Introducción}

El profesorado de Educación Superior debe realizar un seguimiento del aprendizaje del alumnado a partir de tutorías académicas. Estas se caracterizan por la relación existente entre estudiantes y profesorado en los distintos procesos e intercambios que surgen a lo largo de asignatura. Una forma de tutorizar al alumnado es a partir de Proyectos de Aprendizaje Tutorados (PAT), caracterizados por ser una forma de trabajo grupal donde se realiza un aprendizaje de forma autónoma, desarrollando sus competencias profesionales y habilidades, en diferentes escenarios y bajo la supervisión de su profesor o profesora (Álvarez, García, Gil \& Romero, 2004). Según Martínez-Mínguez (2015) y López y Vicente (2015) el PAT es considerado como una "buena práctica”, debido a que son prácticas que facilitan actividades de aprendizaje eficaces y que sirven de ejemplo para otras actividades (Alejandre, 2011).

En el caso de la formación inicial del profesorado, la relación entre el ámbito académico y la escuela es todavía lejana y escasa; se deberían de incorporar perspectivas externas de profesionales reales en el ámbito universitario para así aproximar la teoría y la práctica, a partir de situaciones de aprendizaje en contextos reales. Un PAT dirigido por el profesorado universitario y compartido con docentes de distintas escuelas permite al estudiantado realizar un aprendizaje más vivencial, creando una conexión real entre aquellos conocimientos teórico-prácticos adquiridos en la universidad y los que son claves para la práctica diaria de un docente.

Proyecto de Aprendizaje Tutorado: Sesión de psicomotricidad. Buena práctica para maestrospsicomotricistas de escuelas de infantil y primaria de Barcelona en colaboración con la UAB 
Aunque no debería ser así, a un profesor de universidad le puede ser difícil tutorizar y costoso acercar escenarios de práctica real a sus estudiantes de manera vivencial en asignaturas que no sean Prácticum. En el caso del papel de los docentes de escuela que comparten con el profesorado universitario la tutorización de una asignatura nos preguntamos: ¿qué percepción tienen estos docentes en referencia a su participación en el PAT? ¿consideran que el PAT está bien planificado y organizado para favorecer el aprendizaje del alumnado?

Para resolver estas preguntas se plantea el siguiente objetivo: Analizar las percepciones del docente psicomotricista de escuela en relación a los Proyectos de Aprendizaje Tutorados (PAT), compartiendo con el profesorado universitario su tutorización académica, en una asignatura de formación inicial.

\section{Método}

El estudio se ha llevado a cabo con los docentes psicomotricistas de escuelas que participan en el Proyecto de Aprendizaje Tutorado (PAT), de la asignatura "Educación Psicomotriz en los centros de Educación Infantil” del Grado de Educación Infantil, en la Universidad Autónoma de Barcelona, durante el curso 2016-2017.

El instrumento utilizado ha sido la entrevista semiestructurada, realizada a seis maestros-psicomotricistas de los ocho que colaboran en el proceso de tutoría académica, junto con las profesoras universitarias. En esta investigación se describen las siguientes dimensiones y variables (tabla 1). Los datos se analizaron con el programa de análisis cualitativo NVIVO.

Tabla 1.

Dimensiones y variables de la investigación

\begin{tabular}{ll}
\hline \multicolumn{1}{c}{ DIMENSIONES } & \multicolumn{1}{c}{ VARIABLES } \\
\hline 1. Organizativa- Gestora & 1.1 Informativa \\
& 1.2. Comunicativa \\
& 1.3. Planificadora \\
\hline 2. Pedagógica- Profesional- Didáctica & 2.1. Metodológica \\
& 2.2. Curricular \\
& 2.3. Evaluativa \\
\hline 3. Emocional - Afectiva & 3.1 Interpersonal \\
& 3.2. Intrapersonal \\
\hline
\end{tabular}

A continuación se analizan los datos relacionados con la dimensión organizativa-gestora y la pedagógica-profesional-didáctica del PAT, en concreto se 
describe sobre el aspecto informativo (1.1.), planificativo (1.3.) y evaluativo del PAT (2.3.).

\subsection{Aspecto informativo del PAT}

Los docentes de las escuelas coinciden en la existencia de claridad de la información por parte de las profesoras universitarias. Esta claridad ha facilitado que ellas y ellos conociesen la estructura de la asignatura y pudiesen realizar en sus centros educativos un retorno significativo con el alumnado de prácticas.

"Es un trabajo que está bien estructurado, siempre se nos ha enviado antes de que viniesen los grupos a hacer el primer contacto, los objetivos, la tabla de valoración para que fuésemos viendo un poco qué se debe valorar de las sesiones" (Entrevistado 5).

Por el contrario, algunos docentes describen que no todos los grupos facilitaban toda la información sobre su PAT.

"Yo creo que ellos no eran realmente conscientes de la información que debían traspasar al maestro de escuela" (Entrev. 2)

\subsection{Aspecto de planificación del PAT}

El docente caracteriza la organización de la asignatura como coherente y muy bien elaborada. Una de las características de este proyecto es el trabajo en grupo de las estudiantes; los maestros y maestras coinciden en considerar positivo el hecho de que se realicen agrupaciones de 5 personas. Esta organización les ayuda a compartir y complementar sus individualidades, consiguiendo un resultado más complejo y significativo.

\subsection{Aspecto evaluativo del PAT}

El docente considera favorable el feedback que realizan con las estudiantes, una vez que finalizan la sesión práctica de psicomotricidad. Este feedback permite al estudiantado reflexionar sobre aquellos aspectos de la sesión que no se habían dado cuenta, debido a la complejidad que comporta una práctica de psicomotricidad con niños y niñas entre 0 y 6 años.

No obstante, una maestra percibe la importancia de un mayor conocimiento del proceso previo de las estudiantes ya que este seguimiento permitiría al docente realizar un feedback más específico y significativo. 
"Me gustaría participar y poder ver más su proceso, ver desde que ellas reciben la propuesta, cómo generan y piensan sus ideas; poder hacer un poco este seguimiento que creo que también sería parte del proceso; esto nos daría más pautas a la hora de poder hacer una valoración más detallada” (Entrev. 4)

\section{Conclusiones}

Como conclusiones de esta investigación podemos afirmar que el docente psicomotricista percibe el PAT como una herramienta clave y una buena práctica para la adquisición de un aprendizaje significativo y competencial.

En referencia a la organización del PAT, el papel del profesorado universitario es fundamental para la realización de un proceso tan complejo como es el acercamiento de contextos reales a los y las estudiantes. Por último, el docente psicomotricista considera enriquecedor el feedback realizado con los y las estudiantes ya que les ayuda a reflexionar sobre aspectos específicos que sólo pueden analizarse cuando se está en contacto con una clase real de niños y niñas entre 0 y 6 años.

\section{Referencias}

Alejandre, J.L. (2011). Buenas prácticas en docencia universitaria con apoyo de TIC. Experiencias en 2011. Zaragoza: Prensas Universitarias de Zaragoza.

Álvarez Rojo, V., García Jiménez, E., Gil Flores, J., \& Romero Rodríguez, S. (2004). La enseñanza universitaria: Planificación y desarrollo de la docencia. Almería: EOS.

López, M A A., \& Vicente, F. (2015). Proyecto de aprendizaje tutorado en la formación científica inicial de las estudiantes del Grado Educación Infantil. En N. González, I. Salcines, \& E. García, Tendencias emergentes en evaluación formativa y compartida en docencia. El papel de las nuevas tecnologías. Actas IX Congreso Internacional de Evaluación Formativa y Compartida en Docencia Infantil, Primaria, Secundaria y Universitaria (pp. 959-978). Santander: Universidad Cantabria.

Martínez-Mínguez, L. (2015). Proyecto docente psicomotriz: Buena práctica en el Grado de Educación Infantil de la UAB. En N. González, I. Salcines \& E. García, Tendencias emergentes en evaluación formativa y compartida en docencia. El papel de las nuevas tecnologías. Actas IX Congreso Internacional de Evaluación

Proyecto de Aprendizaje Tutorado: Sesión de psicomotricidad. Buena práctica para maestrospsicomotricistas de escuelas de infantil y primaria de Barcelona en colaboración con la UAB 
Formativa y Compartida en Docencia Infantil, Primaria, Secundaria y Universitaria (pp.1093-1120). Santander: Universidad Cantabria.

Proyecto de Aprendizaje Tutorado: Sesión de psicomotricidad. Buena práctica para maestrospsicomotricistas de escuelas de infantil y primaria de Barcelona en colaboración con la UAB 